\title{
Respuesta del maíz híbrido 4028 a la aplicación de enmiendas orgánicas en un suelo de Córdoba - Colombia
}

\author{
Response of hybrid maize 4028 on the application of organic \\ amendments on soil Córdoba - Colombia.
}

\section{Resposta de milho híbrido 4028 à aplicação de emenda orgânico em um solo de Cordoba - Colômbia.}

\author{
José L. Barrera-Violeth ${ }^{1 *}$; Eliecer M. Cabrales-Herrera" ${ }^{2 *}$; Eliana P. Sáenz-Narváez ${ }^{3}$
}

1 Ing. Agrónomo, MSc, Docente Cultivos, Universidad de Córdoba.
2 Ing. Agrónomo, PhD, Docente área suelos, Universidad de Córdoba.
3 Ing. Agrónomo, Investigador y asistente técnico de cultivos tropicales
*
Grupo de Investigación Agricultura Sostenible, Universidad de Córdoba
Email: jbarrera11@gmail.com

Recibido: 28 de abril de $2015 \quad$ Aceptado: 03 de junio 2017

\begin{abstract}
Resumen
En suelos del Valle del Sinú Medio de mediana fertilidad se evaluó, en un cultivo de maíz hibrido, el efecto de varias fuentes de abonos orgánicos, cuyos tratamientos fueron los siguientes: T1 (Bocashi), T2 (compost con volteos semanales sin microorganismos eficientes (EM), T3 (compost con volteos semanales con EM), T4 (compost con volteos quincenales sin EM), T5 (compost con volteos quincenales con EM), TR (testigo relativo, con NPK en dosis de 100-40-30 kg.ha-1 ${ }^{-1}$, y T0 (testigo absoluto, sin aplicación de abono ni fertilizante). La dosis de abono utilizado fue de 2 t.ha $^{-1}$. Las variables evaluadas fueron rendimiento de grano y crecimiento del cultivo, de las cuales se estimaron los índices fisiológicos (TCA o tasa de crecimiento absoluto, TCR o tasa de crecimiento relativo y TAN o tasa de asimilación neta); el diseño experimental usado fue de bloques completos al azar. Se obtuvo que los mayores rendimientos de grano correspondieron a la fertilización química $\left(6.86 \mathrm{t}\right.$ ha $\left.^{-1}\right)$ y a la fertilización con Bocashi $\left(6.40\right.$ t. ha $\left.{ }^{-1}\right)$ sin diferencias estadísticas significativas entre estos dos tratamientos. Por otro lado, no se encontraron diferencias estadísticas significativas en los parámetros fisiológicos evaluados como altura de planta, número de hojas, masa seca y área foliar. Para este experimento se concluye que el Bocashi puede sustituir la fertilización química bajo las condiciones edafoclimáticas del Valle del Sinú Medio
\end{abstract}

Palabras clave: Bocashi, abonos orgánicos en maíz, organismos eficientes en maíz, índices fisiológicos del maíz.

\begin{abstract}
Abstrac
In soils of Valle de Sinú Medio of medium fertility, the effect of several sources of organic fertilizers was evaluated in a hybrid corn crop, whose treatments were the following:: T1 (Bocashi), T2 (compost with weekly mixture without effective microorganisms EM-); T3(compost weekly mixture with EM); T4 (compost with fortnightly mixture without MS); T5 (compost with fortnightly mixture with MS), T6 at doses 2 t.ha $^{-1}$ for all treatments with organic fertilizers and the absolute check (T0) received no fertilizers, while the relative check (TR) was fertilized with NPK doses of 100-40-30 kg.ha ${ }^{-1}$. The evaluated
\end{abstract}


variables were grain yield and crop growth, of which the physiological indices were estimated (TCA or absolute growth rate, TCR or relative growth rate and TAN or net assimilation rate); the experimental design used was randomized complete blocks. It was found that the highest grain yield corresponded to chemical fertilization (6.86 t ha- 1$)$ and fertilization with Bocashi (6.40 tha-1) without significant statistical differences between these two treatments. On the other hand, no significant statistical differences were found in the physiological parameters evaluated as plant height, number of leaves, dry mass and foliar area. For this experiment it is concluded that the Bocashi can substitute the chemical fertilization under the edaphoclimatic conditions of the Valley of the Middle Sinú.

Keywords: Bocashi, organic fertilizers on maize, efficient organisms (EM) in maize, maize physiological indices.

\section{Resumo}

Em solos de Valle del Sinu Medio de fertilidade média, foi availado o efeito de diversas fontes de fertilizantes orgânicos em uma cultura de milho híbrido, cujos tratamentos foram os seguintes: T1 (Bocashi), T2 (compost semanal misturando sem microrganismos eficazes EM), T3 (compost semanal misturando mais MS), T4 (compost quinsenal misturado sem MS), T5 (compost quinzenal misturado com MS), em doses 2 t ha-1 para todas as tratamentos e tratamento control (T0) sem nenhum fertilizante ou adubos orgânicos, enquanto a tratamento control relativo (TR) só fertilizado com NPK en doses 100-40-30 $\mathrm{kg} \mathrm{ha}^{-1}$. As variáveis avaliadas foram rendimento de grãos e crescimento da cultura da milho, das quais foram estimados os índices fisiológicos (TCA ou taxa de crescimento absoluto, TCR ou taxa de crescimento relativo e TAN ou taxa de assimilação líquida), o delineamento experimental utilizado foi em blocos inteiramente casualizado. Verificou-se que os maiores rendimentos de grãos corresponderam à adubação química (6,86 tha-1) e a adubação com Bocashi $(6,40$ t ha-1), sem diferenças estatisticamente significativas entre os dois tratamentos. Por outro lado, não foram encontradas diferenças estatisticamente significantes nos parâmetros fisiológicos avaliados como altura de planta, número de folhas, massa seca e área foliar. Para este experimento conclui-se que o Bocashi pode substituir a adubação química sob as condições edafoclimáticas do Vale do Médio Sinú.

Palavras claves: Bocashi, fertilizantes orgânicos em milho, microrganismos eficazes em milho, milho índices fisiológicos.

\section{Introducción}

El uso de abonos orgánicos en la agricultura data desde antes de Revolución Verde, con muy buenos resultados y un valor agregado que es la amigabilidad con el medio ambiente, con la que se logra mejorar las propiedades físico-químicas de los suelos y su fertilidad natural, lo que finalmente se ve reflejado en el desarrollo y crecimiento de las plantas, sin embargo, la eficiencia del abono orgánico depende de su origen, edad, composición química y aporte de nutrimentos, entre otros (López-Martínez et al., 2001). La acumulación de materia seca por una población de plantas, depende principalmente del total de carbono fijado y de la intercepción de radiación fotosintéticamente activa, además del uso eficiente por el dosel del cultivo (Sánchez et al., 2012).

Los abonos orgánicos son resultados de diferentes procesos controlados sobre residuos orgánicos, que involucra la acción biológica de microorganismos y que sirve como aporte biológico y aporte nutricional para las plantas (Gómez, 2000). En este sentido, Piccinini et al., (1991), manifiesta que el uso de los abonos orgánicos y su influencia sobre la fertilidad de los suelos ha sido demostrada, aunque su composición química, aporte de nutrimentos a los cultivos y su efecto en el suelo, depende de varios factores, entre los que se resaltan no solo la edad y tipo de material orgánico compostado, sino, el manejo de los residuos, contenido de humedad del suelo y cantidad material orgánico. Se ha demostrado también que los abonos orgánicos pueden influir en minimizar la severidad de patógenos del suelo, como también ser fuente aunque en pequeñas cantidades de nutrimentos para las plantas y mejorador de propiedades fisicoquímicas del suelo (FAO, 1991; Cabrales, 2008).

Los suelos agrícolas por su inadecuado manejo han desmejorado su fertilidad natural, por lo que se ha convertido en un soporte mecánico de baja oferta nutricional, poca retención de nutrientes, en donde la producción agrícola depende de la cantidad de fertilizante que se aplique como complemento en las planes de fertilización; sin embargo, con la aplicación de buenas prácticas de manejo en la que se incluya el uso de materia orgánica al suelo o en su efecto abonos orgánicos, la productividad del suelo puede mejorar e incrementar aún más, cuando se les incorporan organismos eficientes (Vento, 2000; Pérez et al., 2008).

Dentro de las prácticas de manejo de suelos en donde la sostenibilidad es el objetivo principal, los abonos orgánicos son quizás la mejor herramienta en la medida que además de mejorar propiedades físicas del suelo, aporta un valor agregado al mismo, como es el aporte de nutrientes a los suelos agrícolas, aunque su aporte es pequeño, puede contribuir a mejorar la fertilidad natural del suelo (Jeavons 2002; Paneque y Calaña 
2004). Del tipo de abono y su calidad depende el éxito de las mejoras en las propiedades físicas, químicas y biológicas (Lasaridi et al., 2006); y esta calidad puede ser determinada incluso, por el contenido nutricional y la capacidad de proveer nutrientes al suelo y que el cultivo puede aprovechar (Leblanc et al., 2007). Por lo tanto, el aporte nutricional del abono está directamente relacionado con las concentraciones de minerales que poseen los materiales utilizados para su elaboración (Benzing, 2001), además del grado de compostado (Cabrales, 2008). En este sentido, Martínez et al., (2008), evaluaron el compostaje de diversos residuos y recomendaron la posibilidad de reciclar los residuos de cebolla cuando son utilizados en mezcla con materiales de baja relación $\mathrm{C} / \mathrm{N}$. Asimismo, utilizaron estiércol de vacuno y alfalfa alcanzando los mejores valores de autocalentamiento y una mayor eficiencia de conversión de estos residuos en compost, que podrían ser utilizados como enmienda orgánica en agricultura. Al mismo tiempo Shintani et al., (2000) comparó la biomasa verde y las mazorcas de maíz cuando se aplicó de compost, Bokashi, fertilizante químico y un testigo sin aplicación, observando que el bocashi superó al compost y a la fertilización química en un 10 y $20 \%$ respectivamente.

Estudios realizados por Álvarez et al., (2010) y Rincón et al., (2007), en el manejo integrado de la fertilización y abonos orgánicos en cultivos de maíz, sugiere la necesidad de estudiar la dinámica de la mineralización y aporte de nutrimentos durante el ciclo de crecimiento del cultivo, en virtud de que se ha demostrado que se mejoran los rendimientos con bajas dosis de fertilizantes cuando se adicionaron fuentes orgánicas (composta, bocashi y humus de lombriz). Adicionalmente, López et al., (2001), encontraron efectos positivos en la aplicación de abonos orgánicos sobre las propiedades físicas y químicas del suelo y el rendimiento del cultivo de maíz con producciones de hasta 6.05 t.ha ${ }^{-1}$ de grano. Estos mismos autores sugieren dosis de abono orgánico de 20 a 30 t.ha $^{-1}$, con las que se pueden mejorar los rendimientos para el cultivo de maíz en suelos de baja oferta nutricional, sin embargo, se requiere de una baja fertilización complementaria para suplir las necesidades del cultivo.
En consideración a lo anterior, esta investigación tuvo como objetivos la evaluación de dos fuentes de abono orgánico: bocashi y compost obtenido con volteos semanales y quincenales, enriquecidos con microorganismos eficientes, utilizando como planta indicadora el cultivo de maíz hibrido en suelos del Valle del Sinú - Córdoba, en la que se evaluaron variables de crecimiento y producción de grano de este cultivo.

\section{Materiales y métodos}

Localización. La investigación se desarrolló en los lotes experimentales de la Universidad de Córdoba - Colombia, localizada geográficamente a 8052 de latitud Norte y $76^{\circ} 58^{`}$ de longitud Oeste, temperatura promedio anual de $28^{\circ} \mathrm{C}$, humedad relativa media anual del $83 \%$ y 1200 mm anuales de precipitación. Según escala de Holdrige, el lote experimental se encuentra en zona de transición de Bosque Seco Tropical - Bosque Húmedo Tropical, zona agroecológica del Caribe (Palencia et al., 2006). Los suelos evaluados corresponden a Inceptisoles de textura franca-arcillosa, bien drenados y de oferta nutricional, como se puede apreciar en la tabla 1.

Preparación del terreno y siembra. Se realizó con labranza convencional: un pase de arado y dos de rastra liviana. La siembra se hizo con maíz Hibrido Pionner Blanco 4082 a una distancia de $1 \mathrm{~m}$ entre surcos y $0.20 \mathrm{~m}$ entre plantas, para una población final de 55.000 plantas.ha-1, que es la recomendada por la casa comercial y la utilizada en la zona para alcanzar una producción que oscila entre 6-7 t.ha ${ }^{-1}$. Se escogió este hibrido, por el ser uno de los más utilizados en la zona del Valle del Sinú. Las arvenses se manejaron manualmente, y las plagas que se presentaron estuvieron por debajo del umbral de daños que no justificó aplicación o manejo de las mismas.

Tratamientos. El abonado se hizo a los 10 días después de la emergencia (DDE) en una sola dosis, mientras que la fertilización química se hizo aplicando todo el $\mathrm{P}$ y $\mathrm{K}$ en pre-siembra incorporada y el $\mathrm{N}$ se fraccionó en 3 partes: 15 días - 25 y 35 DDE aplicando 30, 40 y $40 \mathrm{~kg} / \mathrm{ha}$ de $\mathrm{N}$. los tratamiento que se evaluaron fueron los siguientes:

Tabla 1. Análisis físico-químicos del lote experimental de la Universidad de Córdoba.

\begin{tabular}{|c|c|c|c|c|c|c|c|c|c|c|c|c|c|c|}
\hline $\mathbf{p H}$ & $\mathbf{M . O}$ & $\mathbf{S}$ & $\mathbf{P}$ & $\mathbf{C a}$ & $\mathbf{M g}$ & $\mathbf{K}$ & $\mathbf{N a}$ & $\mathbf{A l}$ & $\mathbf{C I C}$ & $\mathbf{C u}$ & $\mathbf{F e}$ & $\mathbf{Z n}$ & $\mathbf{M n}$ & TEXTURA \\
\hline $\mathbf{1 : 1}$ & $\mathbf{\%}$ & \multicolumn{1}{|c|}{ (mg.kg-1) } & \multicolumn{8}{|c|}{ (cmol+.kg-1) } \\
\hline 6.43 & 1.3 & 47.8 & 26.3 & 15.0 & 6.5 & 0.88 & 0.13 & - & 22.5 & 3.2 & 24.4 & 4.4 & 112 & Franco arcilloso \\
\hline
\end{tabular}


T0: testigo absoluto, sin ningún tipo de fertilización o abonamiento.

TR: testigo relativo, fertilizado con NPK en dosis de 100-40-30, utilizando como fuentes a la Urea, DAP y $\mathrm{KCl}$, respectivamente;

T1: 2 ton/ha de bocashi producido con residuos orgánicos sólidos y harina de origen vegetal fermentada con microorganismos (bacterias, levaduras, actinomicetos y hongos del género Aspergillus y Penicillium).

T2: 2 ton/ha de abono compostado a partir de residuos sólidos orgánicos, con volteos semanales e inoculación de organismos eficientes (EM).

T3: 2 ton/ha de abono compostado a partir de residuos sólidos orgánicos, con volteos semanales sin inoculación de organismos eficientes (EM).

T4: 2 ton/ha de abono compostado a partir de residuos sólidos orgánicos, con volteos quincenales e inoculación de organismos eficientes (EM).

T5: 2 ton/ha de abono compostado a partir de residuos sólidos orgánicos, con volteos semanales sin inoculación de organismos eficientes (EM).

Índices Fisiológicos. Para la determinación indicies fisiológicos en la planta de maíz, se realizaron evaluaciones de altura de planta (AP), materia seca (MS) y área foliar (AF) hasta la 10 semana después de la emergencia. La cuantificación del AF se hizo por el método del sacabocado, mientras que la MS se obtuvo por secado a $70^{\circ} \mathrm{C}$ por 72 horas. Los índices de crecimiento determinados se hicieron según fórmulas descritas por Radford (1967), citado por Hunt (2003), como se muestra en la Tabla 2, las cuales fueron:

- Tasa de Crecimiento Absoluto (TCA), mide el incremento de la masa seca por unidad de tiempo (g.día $\left.{ }^{-1}\right)$.

- Tasa Relativa del Crecimiento (TCR), expresa el incremento en masa seca en un intervalo de tiempo con relación al peso inicial $\left(\mathrm{g} \cdot \mathrm{g}^{-1} \cdot \mathrm{dí}^{-1}\right)$.
- Tasa de Asimilación Neta (TAN), mide la acumulación de masa seca en función del área foliar por unidad de tiempo.

Rendimientos. El rendimiento del maíz se estimó con base en el peso del grano de cinco mazorcas obtenidas de las hileras centrales de cada parcela de cada tratamiento y se expresó en $\mathrm{kg} \mathrm{ha}^{-1}$, para lo cual se utilizó la siguiente fórmula:

$$
\text { Rto }=(\text { Wparcela })(10.000)^{*} \mathrm{FC}
$$

Donde Rto es el rendimiento, Wparcela es el peso del maíz cosechado en un $\mathrm{m}^{2}, 10.000$ es el área en que se quiere expresar los rendimientos, y FC factor de corrección utilizado que fue de $90 \%$. No se evaluó la humedad, por lo tanto no se tuvo factor de corrección por humedad.

Prueba de campo. La investigación se estableció bajo un diseño de bloques completos al azar (DBCA), con siete tratamientos y tres repeticiones, tomando como unidad experimental una parcela de siete surcos de cinco metros y una distancia de siembra de $20 \mathrm{~cm}$ entre sitios con una planta por sitio. La evaluación se hizo en las hileras del medio para evitar el efecto de borde.

Análisis estadístico. El cultivar se evalúo para determinar si existía diferencia estadística significativa en su rendimiento y otras variables fisiológicas al someterse a diferentes tipos de tratamientos. Los datos fueron tabulados y procesados en tablas en Excel y se utilizó el programa estadístico Statistical Análisis System (SAS) versión 9.1 para el análisis de varianza de los datos y la aplicación de pruebas de comparación de madias Tukey $(\mathrm{P} \leq 0.05)$.

\section{Resultados y discusión}

Indicies fisiológicos en la planta de maíz. No presenta diferencias estadísticas significativas $(P>0,05)$ en las variables evaluadas durante el crecimiento: altura

Tabla 2. Índices fisiológicos de crecimiento determinados en el cultivo de maíz.

\begin{tabular}{|l|l|}
\hline \multicolumn{1}{|c|}{ Índice de crecimiento } & \multicolumn{1}{c|}{ Fórmula para su calculo } \\
\hline Tasa de crecimiento absoluto (TCA) & TCA $=$ msf-msi/tf-ti \\
\hline Tasa de crecimiento relativo (TCR) & TCR=ln msf-lnmsi/tf-ti \\
\hline Tasa de asimilación neta (TAN) & $\mathrm{TAN}=(\mathrm{msf}-\mathrm{msi} / \mathrm{tf}-\mathrm{ti}) \mathrm{x}(\ln$ aff-lnafi/aff-afi) \\
\hline
\end{tabular}

msf: masa seca final; msi: masa seca inicial; tf: tiempo final (días); aff: área foliar final; afi: área foliar inicial; Inaff: logaritmo natural de área foliar final; ti: tiempo inicial (días); Inmsf: logaritmo natural de masa seca final; Inmsf: logaritmo natural de masa seca inicial; Inafi: logaritmo natural de área foliar inicial; af: área foliar 
de planta $(\mathrm{AP})$, número de hojas $(\mathrm{NH})$, masa seca $(\mathrm{MS})$ y área foliar (AF) en la planta de maíz, como se señala en la Tabla 3.

Tabla 3. Comportamiento de las variables de crecimiento: MS (masa seca), AF (área foliar) y AP (altura de planta) del Maíz a los 77 después de emergencia, tratado con abonos orgánicos urbanos.

\begin{tabular}{|c|c|c|c|}
\hline Tratamiento & MS $(\mathbf{g})$ & $\mathbf{A F}\left(\mathbf{c m}^{2}\right)$ & AP $(\mathbf{c m})$ \\
\hline T0 & $1,906 \mathrm{a}$ & $263 \mathrm{a}$ & $1.77 \mathrm{a}$ \\
\hline TR & $2,073 \mathrm{a}$ & $280 \mathrm{a}$ & $1.90 \mathrm{a}$ \\
\hline T1 & $2,034 \mathrm{a}$ & $276 \mathrm{a}$ & $1.85 \mathrm{a}$ \\
\hline T2 & $1,956 \mathrm{a}$ & $263 \mathrm{a}$ & $1.81 \mathrm{a}$ \\
\hline T3 & $1,931 \mathrm{a}$ & $258 \mathrm{a}$ & $1.76 \mathrm{a}$ \\
\hline T4 & $1,917 \mathrm{a}$ & $277 \mathrm{a}$ & $1.86 \mathrm{a}$ \\
\hline T5 & $1,946 \mathrm{a}$ & $281 \mathrm{a}$ & $1.81 \mathrm{a}$ \\
\hline CV & 26,07 & $38,7 \mathrm{a}$ & 6,09 \\
\hline $\mathrm{R}^{2}$ & 0,55 & 0,41 & 0,54 \\
\hline Significancia & $\mathrm{NS}$ & $\mathrm{NS}$ & $\mathrm{NS}$ \\
\hline
\end{tabular}

Letras iguales indican que no hay diferencias estadísticas significativas al 5\%. NS: No significativo; CV: coeficiente de variación; $\mathrm{R}^{2}$. coeficiente de determinación.

La altura promedio a las 21 DDE fue de 17 cm y llegó hasta $175 \mathrm{~cm}$ a las 77 DDE. El mayor incremento de altura de la planta de maíz se presentó en los primeros 52 DDE. La masa seca y área foliar promedia en las primeras 21 DDE fue de $1.47 \mathrm{~g} /$ planta y $225 \mathrm{~cm}^{2}$, respectivamente; la cual aumentó hasta llegar a su máximo valor a los $49 \mathrm{DDE}$, con un área de $281 \mathrm{~cm}^{2}$ (Figura 1). Los mayores valores de área foliar de la planta de maíz fueron reportados por el tratamiento químico en el periodo 21 - 77 DDE. Se puede apreciar la misma tendencia que en el maíz abonado con Bocashi, que entre los 14 y 21 dias de evaluación se obtuvieron los

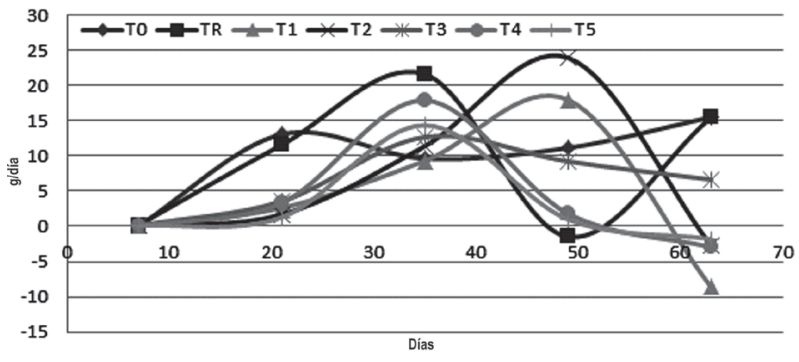

Figura 1. Tasa Absoluta de crecimiento (TAC) de cultivo de maíz bajo diferentes tipos de abonos orgánico de origen urbano y fertilizantes químico. más altos valores, esto se corresponde con los resultados de rendimiento de grano. Estos resultados son similares a los reportados por Jiménez (2010) quien señala que la aplicación de abonos con activadores favoreció su absorción, superando al Bocashi en la producción de gramíneas promisorias. En este sentido Bautista-Cruz et al., (2015) manifiesta que cuando al suelo se le aplica Bocashi, los nutrientes se hacen más disponibles para el cultivo de maíz, lo que puede verse reflejado en un mejor crecimiento de la planta. La Tasa Absoluta de Crecimiento (TAC) del maíz fertilizado con químico y Bocashi, fue similar durante todo el desarrollo del cultivo, como se pudo apreciar en el AF, destacándose una mayor TAC a los 42 días, seguido del maíz tratado con EM de volteos quincenales con y sin inoculación respectivamente, a partir de los $49 \mathrm{DDE}$, se reportaron incrementos de la TAC para todos los tratamientos e inferiores a partir los 56 días en la mayor parte los tratamientos (Figura 1), lo cual se debe posiblemente a que la planta inicia su fase reproductiva. Al respecto, Barrera et al., (2010) señala, que en las etapas vegetativas de las especies anuales la planta produce biomasa de manera eficiente y sostenida en respuesta a la capacidad fotosintética, lo cual explica el comportamiento de este índice de crecimiento que registra altos valores en las primeras etapas del desarrollo, donde la planta de maíz presenta su máxima exposición foliar.

El comportamiento y la tendencia de la Tasa Relativa del Crecimiento (TRC) disminuyeron de manera progresiva para todos los tratamientos. Las curvas observadas en la TRC en el maíz fertilizado con químico y en el que no fue fertilizado con ningún tipo de enmienda fue mayor a los 35 DDE seguido del fertilizado con Bocashi y luego disminuyeron hasta los $42 \mathrm{DDE}$, posteriormente la TRC fue negativa en los maíces fertilizado con químico, sin fertilización y con fertilización a los cuales se le adiciono EM con volteos quincenales e inoculación, ya que la TRC generalmente es alta al inicio y cae al final del ciclo vegetativo, pero se evidencio que el fertilizante químico ofrece mayor cantidad de nutrientes al inicio lo que se traduce en mayor vigor, reflejado en la TRC alta para este tratamiento (Figura 2). En este sentido, Gil y Miranda, (2007) y Bautista-Cruz et al., (2015), afirman que los suelos tratados con abonos orgánicos conllevan a una mejor oferta de nutrientes que pueden favorecer el crecimiento de la plantas, principalmente en sus primeras etapas de desarrollo, presentando mayores TRC en relación a las plantas que se desarrollen en suelos de baja oferta nutricional. Trabajos similares realizados en Alfisoles de baja oferta nutricional corroboran estas afirmaciones, toda vez que los abonos orgánicos en suelos, no solo se espera el aporte nutrimental que pueden poseer, 


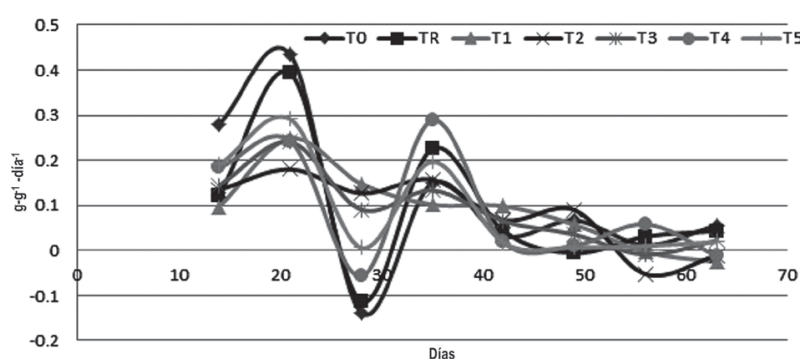

Figura 2. Tasa relativa del Crecimiento (TRC) de cultivo de maíz bajo diferentes tipos de abonos orgánicos de origen urbano y fertilizantes químicos.

sino que enriquecen la biota del suelo y con ello se mejora la capacidad de oferta nutricional, lo cual va a repercutir en el desarrollo del maíz (Daza, 2014).

La Tasa de Asimilación Neta (TAN) del maíz fertilizado con químico y abonado, presentaron un comportamiento similar en el periodo de evaluación de la siembra has 56 DDE y alcanzando su máxima eficiencia a los 35 días, luego a los 42 días mostró un decrecimiento, como se puede apreciar en la figura 3. Los tratamientos restantes, presentaron la misma tendencia de TAN, estos reportaron su mayor eficiencia a los 49 DDE, posteriormente, desde los 66 días la TAN decrece hasta los 70 días, presentando valores negativos para el tratamiento con compost de volteos semanales sin inoculación, como se aprecia en la figura 3. Estos resultados permiten inferir que el maíz bajo las condiciones de crecimiento y desarrollo evaluados, su mayor eficiencia fisiológica se presenta en los primeros 50 DDE, posteriormente disminuye, posiblemente a las condiciones del deterioro del área foliar y el autosombriamiento generado por el traslape de las hojas, que inciden en la intercepción de la radiación, además en la fase productiva la mayor cantidad de asimilados se dirigen hacia la mazorca, órgano que atrae el mayor flujo de asimilados de la hoja bandera principalmente. En este sentido, Hunt (2003) indica, que la TAN exhibe en general un nivel constante relativo, modificado por fluctuaciones en el ambiente durante el período de crecimiento; a esta afirmación se le unen Montemayor et al., (2006), quienes exponen que durante el ciclo de crecimiento y desarrollo de la planta de maíz, la posición de las hojas cambian su arquitectura de erectófilas a planófilas en la mayoría de los casos y, este factor incide directamente en la intercepción de la radiación fotosintéticamente activa, por lo tanto, la posición final que asuma la hoja puede afectar la producción del cultivo de maíz.

Rendimiento del maíz. El análisis estadístico mostró que hay diferencias estadísticas significativas entre el

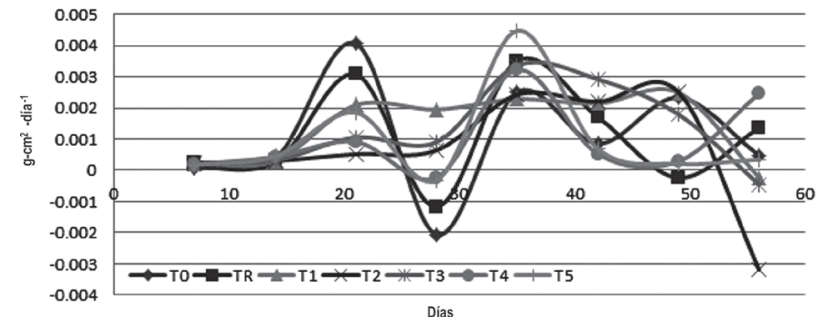

Figura 3. Tasa de asimilación neta TAN del cultivo de maíz bajo diferentes tipos de fertilización de origen urbano y fertilizante químico.

testigo y los demás tratamientos (Figura 4). Se pudo observar que la fertilización química presentó el rendimiento promedio numéricamente más alto de grano $\left(6.86 \mathrm{t} \mathrm{ha}^{-1}\right)$, siguiendo, en orden de importancia, el Bocashi (6.40 $\left.\mathrm{t} \mathrm{ha}^{-1}\right)$, entre estos dos tratamientos no hubo diferencias estadísticas significativas, lo que hace suponer que el abono orgánico tipo Bocashi de cierta manera puede sustituir la fertilización de síntesis química obteniendo rendimiento similar cuando se aplican fertilizantes de sisntesis química. Por otro lado, se pudo demostrar que el uso de Bocashi con volteos cada semana o dos semana, supera el tratamiento testigo a quin no se le aplicó ningún tipo de fertilizante o abono. Estos resultados son similares a los reportados por López-Martínez (2001), quienes obtuvieron mayores rendimientos de maíz en el tratamiento químico, seguido por el de composta. Resultados similares reportan Quintanilla et al., (2013) quienes no encontraron diferencias entre bocashi y fertilización química en la producción de maíz (980 kg/ha) en suelos de baja oferta nutricional, por lo que atribuyen a este bajo rendimiento posiblemente a la baja oferta química del suelo; así mismo Lok y Suarez (2014), había reportado que el estiércol de bovinos incrementó la producción de sorgo, igualmente ensayos agronómicos en cultivo de maíz, abonados con material compostado mostraron que el producto promueve mayor peso de materia seca del maíz que el fertilizante químico (Daza, 2014), lo cual se debe posiblemente a que el uso de los abonos orgánicos enriquecen la biota edáfica y con ello se mejora la capacidad de oferta nutricional del suelo y por ello se incrementa la producción del cultivo de maíz, sin embargo si los suelos son fértiles, la adición de abonos orgánicos pueden no arrojar resultados de significancia estadística (Daza, 2014).

\section{Conclusiones}

Con el uso de abonos orgánicos en el cultivo de maíz en dosis de 2 t/ha en suelos de Valle del Sinú Medio, 


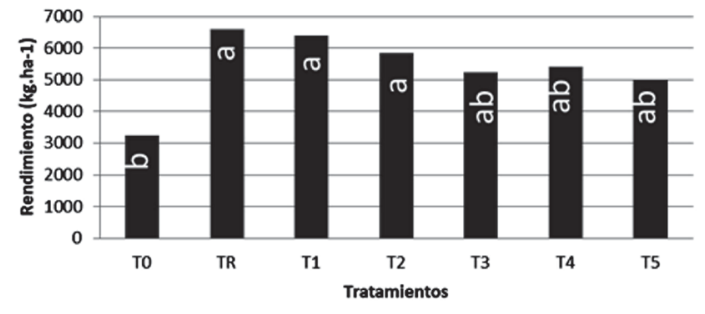

Figura 4. Rendimiento en kg.ha-1 en el cultivo de maíz bajo diferentes tipos de abonos orgánicos de origen urbano y fertilizantes químicos.

los índices fisiológicos del cultivo (TAC, TRC y TAN) no se vieron afectados.

El uso de abono orgánico tipo Bocashi en dosis de 2 ton/ha enriquecido con microorganismos eficientes nativos puede mejorar los rendimientos del cultivo del maíz en condiciones del Medio Sinú.

Los abonos orgánicos tipo Bocashi y compost con volteos semanales o quincenales e inoculados con microorganismos eficientes (EM) nativos de estos suelos, puede ser una alternativa a tener en cuenta en los planes de manejos de suelos en el Valle del Sinú Medio.

Si se tratase de escoger el mejor tipo de abono orgánico, Bocashi podría ser la mejor alternativa para el manejo de suelos en el Vale del Sinú Medio.

Los abonos orgánicos podrían ser una alternativa para la sustitución parcial de la fertilización química (NPK) bajo las condiciones del Valle del Sinú Medio, en la medida que se enriquece el suelo biológicamente y eso puede mejorar la disponibilidad de nutrientes en el mismo.

\section{Agradecimientos}

Al biólogo Ernesto José Martínez por su contribución y apoyo científico en esta investigación y a la Universidad de Córdoba por el financiamiento de la misma.

\section{Referencias}

Álvarez D, Gómez A, León S, Gutiérrez F. Manejo integrado de fertilizantes y abonos orgánicos en el cultivo de maíz. Agrociencia. 2010;44:575-586.

Barrera J, Suárez D, Melgarejo L. 2010. Análisis de crecimiento de las plantas. Experimentos en fisiología Vegetal. Bogotá: Universidad Nacional de Colombia. Primera Edición. 96p.

Bautista-Cruz A, Cruz G, Rodríguez-Mendoza María. Efecto de bocashi y fertilizantes de liberación lenta en algunas propiedades de suelos con maíz. Revista Mexicana de Ciencias Agrícolas. 2015;6(1):217-222.
Benzing A. 2001. Agricultura Orgánica - fundamentos para la región andina. Alemania: Neckar-Verlag, Villingen-Schwenningen. 682 p.

Cabrales E. 2008. Materia orgánica del suelo: material didáctico para universitarios. Montería: Centro de publicaciones Universidad de Córdoba. 65p.

Daza M. Application of waste flowers compost in acid soils cultivated with corn (Zea mays). Revista Científica Técnica Agropecuaria. 2014;23(3):22-30.

FAO. 1991. Manejo del suelo: producción y uso del compostaje en ambientes tropicales y subtropicales. Italia, Boletín 56. 180p.

Hunt R. 2003. Plant growth analysis: individual plants. En: Thomas B, Murphy DJ, Murray D (Eds.). Encyclopedia of applied plant sciences. Academic Press, London, pp 579-588.

Jeavons J. 2002. Cultivo biointensivo de alimentos. Ecology actions of the Midpeninsula. Estados Unidos. 261 p.

Laffite H. Fisiología del maíz tropical. Programa de maíz CIMMT. Tecnifenalce, 2002;2(7):4-5.

Lasaridi K, Protopapa I, Kotsou M, Pilidis G, Manios T, Kyriacou A. Quality assessment of composts in the Greek market: The need for standards and quality assurance. Journal of Environmental Management. 2006;80:58-65.

Leblanc H, Cerrato M, Miranda A, Valle G. Determinación de la calidad de abonos orgánicos a través de bioensayos. Tierra Tropical. 2007;3:97-107.

Castellanos JZ, Márquez JJ, Etchever JD, Aguilar A, Salinas JR. Efecto de largo plazo de la aplicación de estiércol de ganado lechero sobre el rendimiento de forrajes y las propiedades del suelo en una región árida irrigada del norte de México. Terra 1996;14(2):151-158.

López J, Díaz A, Martínez E, Valdez R. Abonos orgánicos y su efecto en propiedades físicas y químicas del suelo abonos orgánicos y su efecto en propiedades físicas y químicas del suelo y rendimiento en maíz. Terra 2001;19:293-299.

López-Martínez J, Díaz A, Martínez E, Valdez R. Abonos orgánicos y su efecto sobre las propiedades físicas y químicas del suelo y el rendimiento del maíz. Terra 2001;19(4):293-300.

Maddonni, G., Cirilo, A., Otegui, M. 2006. Row width and maize grain yield. Agronomy Journal 98(6): 1532-1543.

Martínez R, Miglierina A, Luna M, Van A, Pellerejo G. Evaluación del compostaje de los residuos del procesamiento de la cebolla. Revista Pilquen - Sección Agronomía. 2008;9: 1-8.

Montemayor J, Zermeño A, Olague J, Aldaco R, Fortis M, Salazar E, Rodríguez J, Vázquez C. Efecto de la densidad y estructura del dosel de maíz en la penetración de la radiación solar. International Journal of experimental botany. 2006;75:47-53.

Palencia G, Mercado T, Combatt E. 2006. Estudio Agroclimático del Departamento de Córdoba. Montería: Universidad de Córdoba. $126 p$

Paneque V, Calaña J. 2004. Abonos Orgánicos, conceptos prácticos para su evaluación y aplicación. Folleto Técnico. La Habana: Asociación Cubana de técnicos Agrícolas y forestales. 54 p. 
Pérez A, Céspedes C, Núñez P. Caracterización física-química y biológica de enmiendas orgánicas aplicadas en la producción de cultivos en República Dominicana. Revista de la Ciencia del Suelo y Nutrición vegetal, 2008;8(3):10- 29.

Piccinini S, Bortone G. The fertilizer value of agriculture manure: simple rapid methods of assessment. J. Agric. Eng. Res. 1991;49:197-208.

Quintanilla F, Yanes C, Monge C. 2013. Incidencia del Bocashi, Gallinaza y su combinación con fertilizantes químicos en la mejora de la fertilidad del suelo y en los rendimientos de maíz (Zea mays L.), San Juan Opico, La Libertad. Trabajo de Grado, Ingeniero Agrónomo, Universidad de El Salvador, Facultad Ciencias Agronómicas. 123p.

Rincón A, Ligarreto G, Sanjuanelo D. Crecimiento del maíz y los pastos (Brachiaria sp.) establecidos en monocultivo y asociados en suelos ácidos del piedemonte llanero colombiano. Agronomía Colombiana. 2007;25(2):264-272.

Sánchez D, Ligarreto G, Leiva F. Variabilidad del Crecimiento y Rendimiento del Cultivo de Maíz para Choclo (Zea mays L.) como Respuesta a Diferencias en las Propiedades Químicas del Suelo en la Sabana de Bogotá, Colombia. Rev. Fac. Nal. Agr. 2012;65(2): 6579-6583.

Shintani M, Leblanc H, Tabora P. 2000. Bokashi (Abono Orgánico Fermentado) Tecnología Tradicional Adaptada para una Agricultura Sostenible y un Manejo de Desechos Modernos. Guía para uso práctico. EARTH Guacimo, Limón, Costa Rica. 25p

Vento M. 2000. Estudio sobre la preparación del compost estático y su calidad. Tesis Master en Fertilidad del Suelo, Universidad de Camaguey - Instituto de suelos. Cuba. 